Check for updates

Cite this: RSC Adv., 2017, 7, 50591

\title{
Micro heat pipe device utilizing extended nanofluidics $\dagger$
}

\author{
Chenxi Wang, (D) *a Yutaka Kazoe, (D) ${ }^{\mathrm{b}}$ Kyojiro Morikawa, (D) ${ }^{\mathrm{b}}$ Hisashi Shimizu, (D) ${ }^{\mathrm{b}}$ \\ Yuriy Pihosh, (D) ${ }^{\mathrm{b}}$ Kazuma Mawatari ${ }^{\mathrm{D}}{ }^{\mathrm{b}}$ and Takehiko Kitamori $\left(\mathbb{D}{ }^{* \mathrm{~b}}\right.$
}

\begin{abstract}
The next-generation cooling devices are gradually being scaled to smaller than the size of highperformance microchips to enable local heat removal from small hot spots. Realization of a micro/ nanofluidic heat pipe device is a challenging task, as it requires high condensation efficiency in an ultra-small space and sufficient liquid transport without employing any wick. Herein, we demonstrate a two-phase loop micro heat pipe device based on unique liquid properties in extended nanospace $(10-1000 \mathrm{~nm})$ to meet the growing demands of the miniaturization of electronics and optoelectronics. The device, which contains a small volume of liquid (tens of nanoliter) and does not require a wick, can be conveniently embedded in the microchip. The capillary condensation of water on nanopillars was investigated. The experimental results showed a significant enhancement of the condensation rate on nanopillars for a faster vapor-liquid phase transition. In addition, a streaming potential measurement was performed to evaluate the liquid transport during operation of the micro heat pipe device. This method enables the measurement of water flow rates through extended nanochannels without requiring probe molecules. The micro heat pipe device was verified to work properly. Finally, the cooling performance of the micro heat pipe device was quantitatively estimated, and improvements were proposed to achieve highly efficient cooling.
\end{abstract}

Received 8th September 2017 Accepted 26th October 2017

DOI: 10.1039/c7ra10017e

rsc.li/rsc-advances transfer of electronic devices, such as computers, laptops, telecommunication and satellite modules. ${ }^{\mathbf{1 2 , 1 3}}$ However, conventional heat pipes combined with large electric fans are difficult to integrate into microchips. Micro-grooved heat pipes, one of the most commonly used micro heat pipes, are suitable for small semiconductor devices because they can be fabricated and integrated into the microchips through microelectromechanical (MEMS) technology. ${ }^{\mathbf{1 4 1 1 5}}$ In most microgrooved heat pipes, the liquid-vapor friction interactions originating from the countercurrent flow in the same groove can degrade the heat transfer performance. Consequently, twophase loop micro heat pipe devices have emerged as a desirable solution since the liquid and vapor flow paths are separated to avoid the interfacial friction interactions. ${ }^{16,17}$ Although these two-phase loop devices can sustain a high heat flux, they generally require wicks for operation, which limits their ability to follow the trends of further device miniaturization. To obtain high heat transfer for high-performance wearable devices, it is desirable to embed micro heat pipes in the microchip using very small hydraulic diameters without requiring wicks.

Recently, microfluidic spaces have been scaled down to sizes on the order of 10-1000 $\mathrm{nm}$, which is a range known as an "extended nanospace" to distinguish it from a nanospace in the 1-10 nm range. ${ }^{18}$ Unique liquid properties in extended nanospaces have been reported, e.g., high viscosity, low dielectric constant, and high proton mobility. ${ }^{\mathbf{1 9 - 2 1}}$ Based on these unique
State Key Laboratory of Welding and Joining, Harbin Institute of Technology, Harbin 150001, China. E-mail: wangchenxi@hit.edu.cn; Fax: +86-451-86416186; Tel: +86 451-86418359

${ }^{b}$ Department of Applied Chemistry, School of Engineering, The University of Tokyo, 7-31 Hongo, Bunkyo, Tokyo 113-8656, Japan. E-mail: kitamori@icl.t.u-tokyo.ac.jp; Fax: +81-3-5841-6039; Tel: +81-3-5841-7231

$\dagger$ Electronic supplementary information (ESI) available: Supplementary movie. See DOI: 10.1039/c7ra10017e 
properties, a femtoliter-scale ( $\left.\mathrm{fL}=10^{-15} \mathrm{~L}\right)$ immunoassay $^{22}$ and femtoliter to attoliter-scale $\left(\mathrm{aL}=10^{-18} \mathrm{~L}\right)$ chromatography $^{23}$ platforms have been developed for innovative biomedical and chemical applications, respectively. An autonomous solar-lightdriven micro fuel-cell device has also been realized. ${ }^{24}$ Hibara et al. found that the vapor pressure in extended nanopillars is lower than that on flat surfaces, ${ }^{25}$ suggesting that the capillary condensation of water can be more easily accomplished in extended nanospaces than in bulk spaces. Utilization of the unique properties of water in the extended nanofluidic space can create new possibilities for the development of micro heat pipe devices.

Herein, we propose a two-phase loop micro heat pipe device utilizing extended nanopillars and nanochannels. The device consist of micro/nanochannels that do not incorporate wicks. To address the condensation efficiency in extended nanospaces, the capillary condensation of water on nanopillars is investigated in this study. However, monitoring liquid transports in the extended nanochannels of the micro heat pipe is a tremendous challenge because nanoparticles or fluorescent dyes are not allowed into the liquid-vapor phase. To overcome this problem, we develop a streaming potential measurement to evaluate liquid transports during the operation of the micro heat pipe device. This method enables the measurement of water flow rates in extended nanochannels without requiring probe molecules. Thus, the cooling performance is quantitatively estimated, and improvements to this performance are discussed.

\section{Principle}

Fig. 1 illustrate the conceptual design of the micro heat pipe device utilizing extended nanopillars and nanochannels. The device focuses on local cooling of a hot spot $(\sim \mathrm{mm}$ size $)$ in a several-centimeter-sized VLSI chip. The two-phase loop micro heat pipe consists of a microchannel (for vapor transport) and extended nanochannels on two sides (for liquid transport). The microchannel connected to the two nanochannels is bridged by the extended nanopillars. At the initial stage, nanochannels on both sides are charged with water. When the water is locally heated from the hot spot (i.e., the evaporator), the generated vapor flows along the microchannel to the nanopillars (i.e., the condenser), allowing enhanced capillary condensation of water due to the unique liquid properties in extended nanospace. After water condensation occurs, the high Laplace pressure dominated by the surface tension in the side nanochannels can drive the condensation water back to the evaporator for reevaporation. The liquid-vapor circulates in a closed-loop micro/nanospace, while evaporation and condensation occur simultaneously for heat absorption and dissipation, respectively. The capillary condensation efficiency and the liquid transport play two important roles in the cooling performance.

\section{Experimental}

\subsection{Device design and fabrication}

Extended nanopillars (pillar width $=$ pillar interval) were fabricated on fused silica substrates (VIOSIL-SQ, Shinetsu Chemical, Japan) by electron beam lithography and plasma etching. The fabrication process of the nanopillars is similar to the nanochannel fabrication process described in the literature. ${ }^{26}$ The extended nanopillars with various intervals ranging from $150 \mathrm{~nm}$ to $2000 \mathrm{~nm}$ were successfully fabricated and were confirmed using scanning electron microscopy (SEM). The depth of all the nanopillars was $300 \mathrm{~nm}$. An investigation of the capillary condensation of water on the nanopillars was performed.

Fig. 2 shows the design and a photograph of the micro heat pipe device, which includes the vapor condensation and the condensation water circulation. On one fused silica substrate, a microchannel (with a $1000 \mu \mathrm{m}$ width and a $3 \mu \mathrm{m}$ depth) was fabricated to transport the vapor from an evaporator measuring $1 \mathrm{~mm}^{2}$. On another substrate, a two-dimensional extended nanopillar array (with a $250 \mathrm{~nm}$ interval and a $300 \mathrm{~nm}$ depth) was fabricated, which was proposed as a condenser to allow enhanced capillary condensation. Meanwhile, two sets of side extended nanochannels (with a $500 \mu \mathrm{m}$ width and a $300 \mathrm{~nm}$ depth) that were connected to the nanopillars were fabricated to

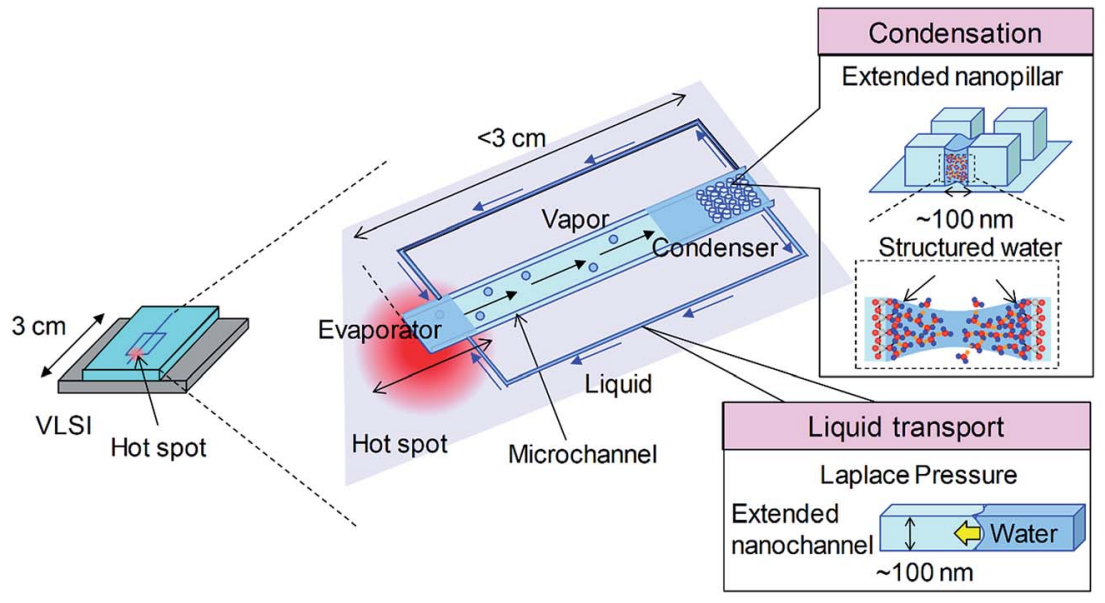

Fig. 1 Conceptual design of the micro heat pipe device utilizing extended nanopillars and nanochannels. 


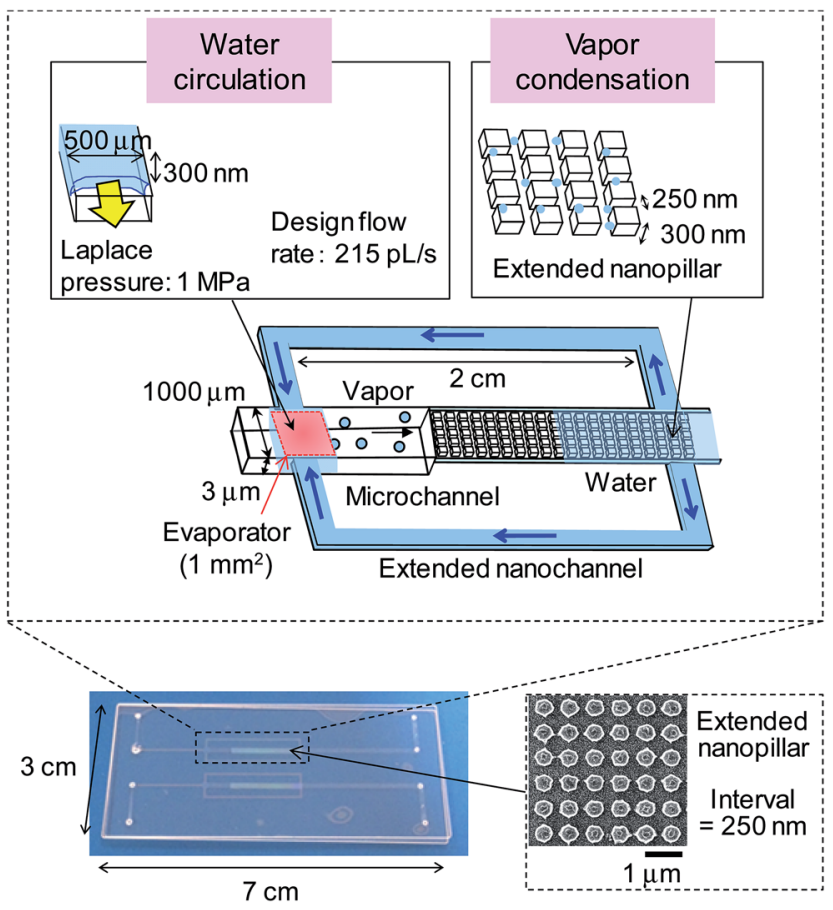

Fig. 2 Design parameters and photograph of the micro heat pipe device. An inset SEM image shows the extended nanopillars with a $250 \mathrm{~nm}$ interval integrated in the device.

transport the condensation water. The two fused silica substrates were finally bonded using a low-temperature bonding process at $110{ }^{\circ} \mathrm{C} .{ }^{27}$

During device operation, the capillary flow occurs spontaneously, and the flow rate depends on the balance between the Laplace pressure $P_{\mathrm{L}}$ (driving force) and the pressure drop $P_{\mathrm{f}}$ (friction drag). From the Young-Laplace equation and the Hagen-Poiseuille law, the flow rate can be calculated as follows: ${ }^{28}$

$$
\begin{gathered}
P_{\mathrm{L}}=\frac{2 \gamma \cos \theta}{r}=\frac{4 \gamma \cos \theta}{D_{\mathrm{h}}} \\
P_{\mathrm{f}}=\frac{128 \mu L}{\pi D_{\mathrm{h}}{ }^{4}} Q
\end{gathered}
$$

where $\gamma$ is the surface tension, $\theta$ is the liquid contact angle, and $D_{\mathrm{h}}$ is the hydrodynamic diameter, which is equal to the nanochannel depth $(=300 \mathrm{~nm})$ for the plate channel in our case. The parameter $\mu$ is the liquid viscosity, $L$ is the extended nanochannel length, and $Q$ is the flow rate. Considering these parameters, we calculated a Laplace pressure $P_{\mathrm{L}}$ of $\sim 1 \mathrm{MPa}$ for driving the liquid, and the design flow rate that can be driven in one extended nanochannel is $107 \mathrm{pL} \mathrm{s}^{-1}\left(215 \mathrm{pL} \mathrm{s}^{-1}\right.$ for two sets in total) in the case of $P_{\mathrm{L}}=P_{\mathrm{f}}$.

\subsection{Heat pipe device working system}

After the micro heat pipe device was fabricated, it was partially filled with deionized (DI) water. Prior to charging the device with water, non-condensable air in the device must be removed because the residual air may degrade the device performance.
Therefore, the device should be evacuated to a low pressure. Fig. $3 \mathrm{a}$ and $\mathrm{b}$ illustrate a vacuum system including the gas lines that we developed to evacuate the residual air from the micro/ nanochannels. The device was integrated with a stainless chip holder in which the gas/water inlets and outlets were sealed hermetically. The pressure of the vacuum background was $\sim 0.3$ $\mathrm{kPa}$ before introducing water. The DI water was degassed initially and then was introduced into the designated micro/ nanochannels using a controlled pressure of Ar gas. The working environment was maintained at room temperature $\left(19 \pm 1^{\circ} \mathrm{C}\right)$. A specific temperature control stage (Citizen, Japan) was employed to accurately heat the device at the evaporator position (evaporator size: $1 \times 1 \mathrm{~mm}^{2}$ ) over a range from $19^{\circ} \mathrm{C}$ to $120{ }^{\circ} \mathrm{C}$. Additionally, five sets of micro thermocouples (not shown in Fig. $3 \mathrm{~b}$ ) were attached to the device to measure the temperatures at various positions.

\subsection{Flow rate measurement system}

To verify the device working principle and to evaluate the cooling performance, the flow rates in the extended nanochannels were monitored by the streaming potential

(a)

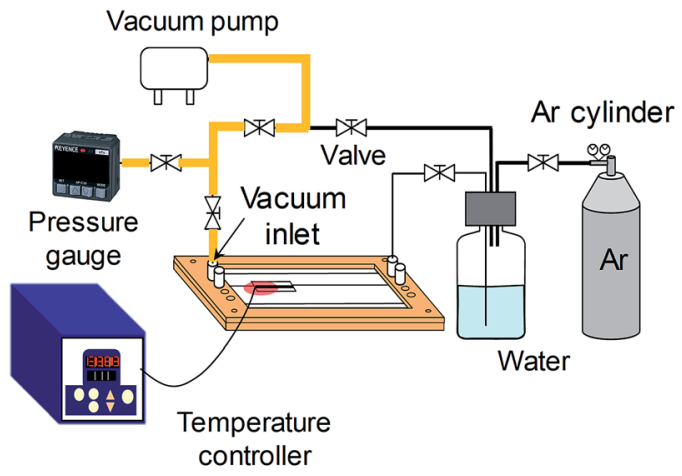

(b)

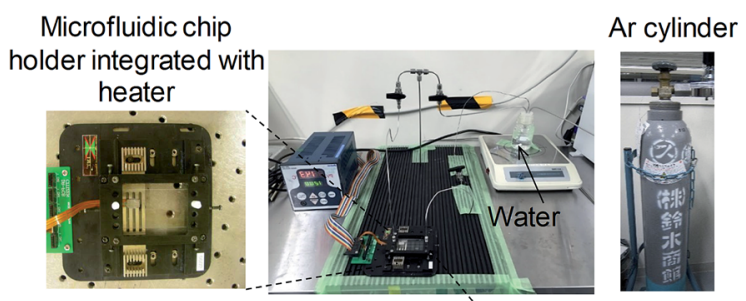

(c)

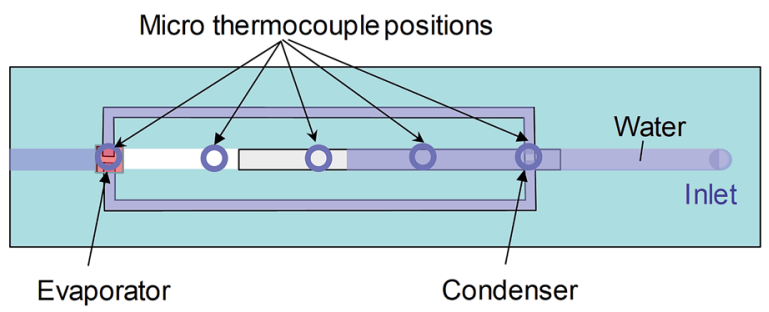

Fig. 3 (a) Schematic illustration of the vacuum system to evacuate the residual gases from the micro/nanochannels and from the gas lines (the orange color). The deionized (DI) water was then charged into designated micro/nanochannels using a controlled pressure of Ar gas. (b) Photograph of the experimental setup. (c) Temperature measurements using micro thermocouples on the micro heat pipe device. 

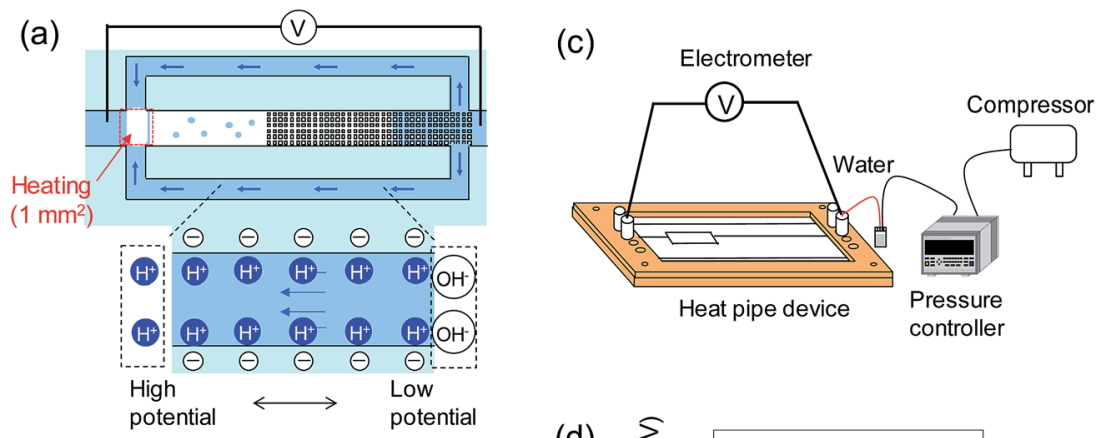

(b)

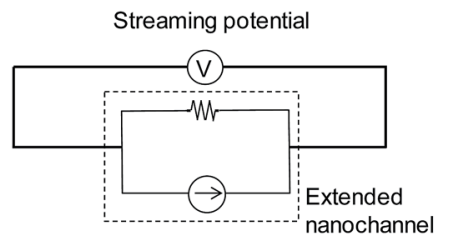

(d)

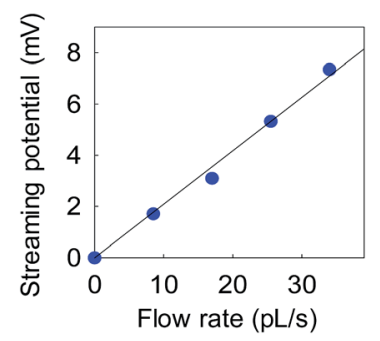

Fig. 4 (a) Schematic illustration of the streaming potential measurement for monitoring the flow rate in the extended nanochannel during operation of the heat pipe device and the equivalent circuit (b). In the calibration test, the DI water flow was driven into the extended nanochannel by a pressure controller (c), and the calibration curve was constructed by plotting streaming potential against flow rate (d).

measurement. The detailed principle of the streaming potential/current measurement has been previously reported. ${ }^{29,30}$ Fig. 4a illustrates monitoring of the flow rate in the extended nanochannels while the heat pipe device was operating. For electrical measurements, $\mathrm{Ag} / \mathrm{AgCl}$ electrodes (fabricated by coating Pt wires with $\mathrm{Ag} / \mathrm{AgCl}$ paste) were inserted into the inlet and the outlet reservoirs of the microchannels. Each electrode was connected to an electrometer (Keithley 6514, Keithley Instruments, USA) using voltage measurement mode. The equivalent circuit is shown in Fig. 4b. The microchannel between the evaporator and the condenser is an electrical disconnection because of the vapor path. In the calibration test, the DI water flow was driven in the extended nanochannel by a pressure controller (Fig. 4c), and the streaming potential generated between $\mathrm{Ag} / \mathrm{AgCl}$ electrodes was detected by the electrometer. The noise generated in the connected microchannels could be ignored. The calibration curve was constructed by plotting streaming potential against flow rate in the extended nanochannel (Fig. 4d).

\section{Results and discussion}

\subsection{Capillary condensation on nanopillars}

The capillary condensation of water on extended nanopillars was characterized. Four sets of nanopillar arrays with different intervals, e.g. $2000 \mathrm{~nm}, 1000 \mathrm{~nm}, 500 \mathrm{~nm}$ and $250 \mathrm{~nm}$, were fabricated on a fused-silica glass substrate as a group. Fig. 5a shows the experimental setup. The substrate with the fabricated nanopillars was fully attached to a transparent glass cover, which maintained the DI water on a temperature control stage for evaporation without outside leakage. To avoid an undesirable heat effect during the measurement, an optical microscope (Nikon E400, Nikon, Japan) with a UV-filtered light source was employed to record the capillary condensation process using a charge-coupled device (CCD) camera. The experimental environment was maintained at room temperature $\left(19 \pm 1{ }^{\circ} \mathrm{C}\right)$.

When the water was evaporated (the temperature was elevated to $35{ }^{\circ} \mathrm{C}$ at the stage), the generated vapor moved upward and condensation occurred on the four sets of nanopillars simultaneously. Fig. 5b illustrates the capillary condensation process, which consisted of the nucleation and filling of the nanopillar intervals. The reflected light intensity of the nanopillar region decreased during the condensation process due to the interference between the reflected lights. The CCD captured micrographs of the $250 \mathrm{~nm}$ nanopillars changing from light to dark during capillary condensation, as shown in Fig. 5c (see the real-time movie in the ESI†). Imaging processing software was employed to quantitatively analyze the condensation region. In our experiments, the condensation water always filled the small-sized nanopillars before filling the large-sized ones. The capillary condensation rate was calculated by the ratio of the condensation water volume to the condensation time.

Fig. $5 \mathrm{~d}$ shows the size dependency of the capillary condensation rate of water on nanopillars. The condensation rate increased significantly with decreasing nanospace size. In particular, the condensation rate on $150 \mathrm{~nm}$ nanopillars was much higher ( $>3$ times higher) than the rate on $1000 \mathrm{~nm}$ nanopillars. Due to the effect of the surface-to-volume ratio, the total surface area of small-sized nanopillars is larger than that of large-sized ones. Therefore, water molecules have more opportunities for nucleation and for bridging easily during the water filling process into the intervals. In addition, in this extended nanospace, the properties of uniquely structured water with high proton mobility and high viscosity were reported in our previous study. ${ }^{19,21}$ This enhanced condensation in an extended nanospace is considered to facilitate a faster vapor-liquid phase transition. 


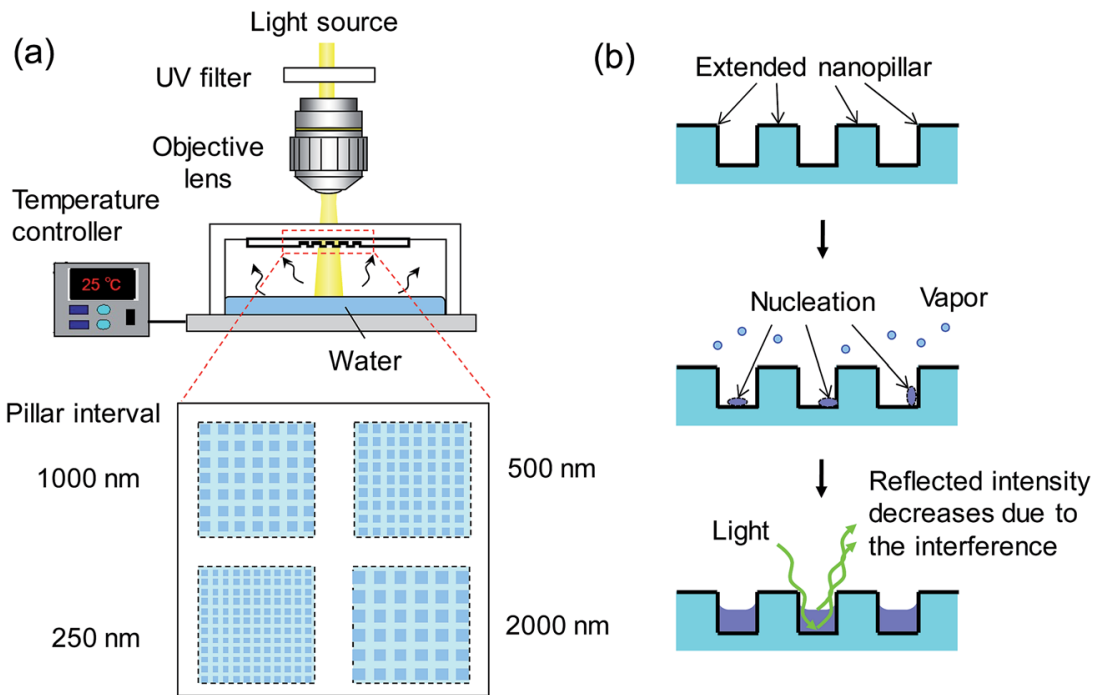

(c)

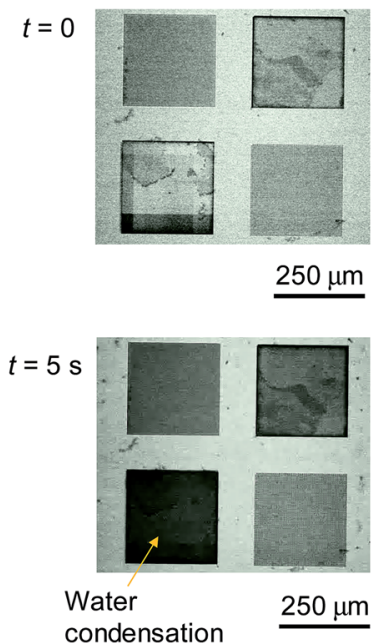

(d)

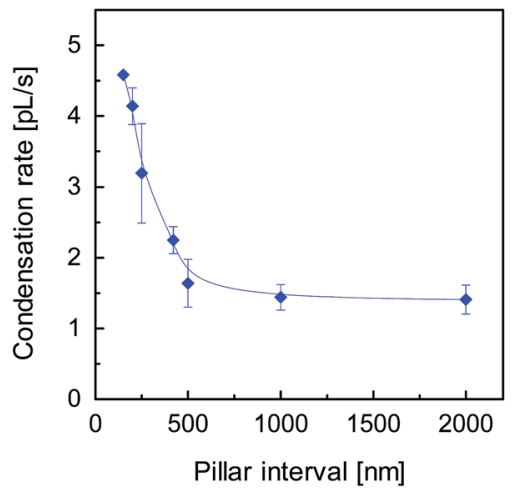

Fig. 5 Characterizations of capillary condensation of water on extended nanopillars. (a) Experimental setup; (b) schematic illustrations of the capillary condensation process; (c) optical micrographs before and after the capillary condensation; and (d) size dependency of the capillary condensation rate of water on extended nanopillars.

\subsection{Verification of the device working principle}

The flow rate of the condensation water in the extended nanochannels (returning to the evaporator) was monitored to verify proper operation of the heat pipe. During the device operation, the liquid/vapor flow was autonomous in all micro/ nanochannels without applying any external pressure. The evaporator was heated and maintained at $54{ }^{\circ} \mathrm{C}, 80{ }^{\circ} \mathrm{C}$ and $110{ }^{\circ} \mathrm{C}$ for $120 \mathrm{~min}$ at each temperature. Each elevated temperature at the evaporator was calibrated using the micro thermocouple. Fig. 6 shows the water flow rates in the extended nanochannel as monitored by the streaming potential measurements. At the initial stage, all the water flow occurred in a reverse direction due to the evaporation and resistancecapacitance (RC)-circuit delay. As shown in Fig. 6a, the evaporator was maintained at $54{ }^{\circ} \mathrm{C}$ for $120 \mathrm{~min}$, and the flow rate was $40 \mathrm{pL} \mathrm{s}^{-1}$. During the operation, the accumulated water volume along the extended nanochannels was $\sim 248 \mathrm{~nL}$. The design water volume in the two extended nanochannels was $15 \mathrm{~nL}$. The results demonstrate that more than 16 cycles of water circulation occurred in $120 \mathrm{~min}$, confirming that the micro heat pipe device operated properly. Furthermore, we tested this device could work properly for a longer duration (e.g., $12 \mathrm{~h}$ or more) with no problem.

Fig. 7 shows the water flow rate in the extended nanochannel as a function of evaporator temperature maintained during operation of the heat-pipe device. In general, the water flow rates along the side extended nanochannels increased as the evaporator temperature increased. When the evaporator was heated and maintained at a maximum temperature of $110^{\circ} \mathrm{C}$ for $120 \mathrm{~min}$, the flow rate increased to $150 \mathrm{pL} \mathrm{s}^{-1}$, and more than 56 cycles of the water circulation occurred while the device was operating. For heating at a higher temperature $\left(e . g\right.$., $\left.115^{\circ} \mathrm{C}\right)$, the measured streaming potentials decreased substantially to $\sim 0 \mathrm{mV}$ due to "dry out" at the evaporator. 
(a)

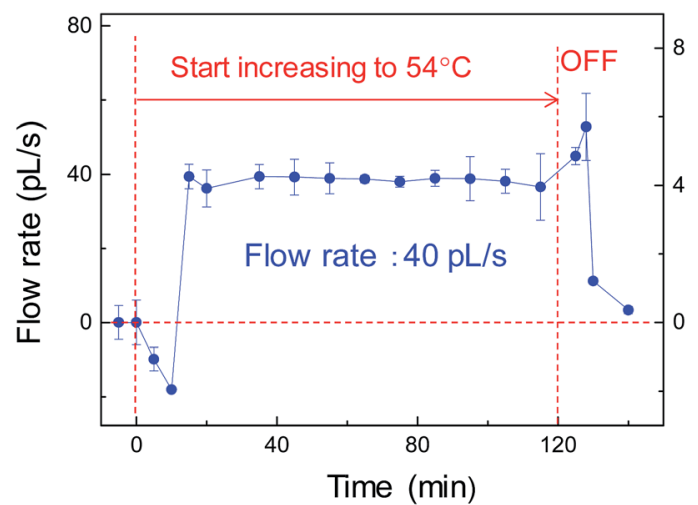

(b)

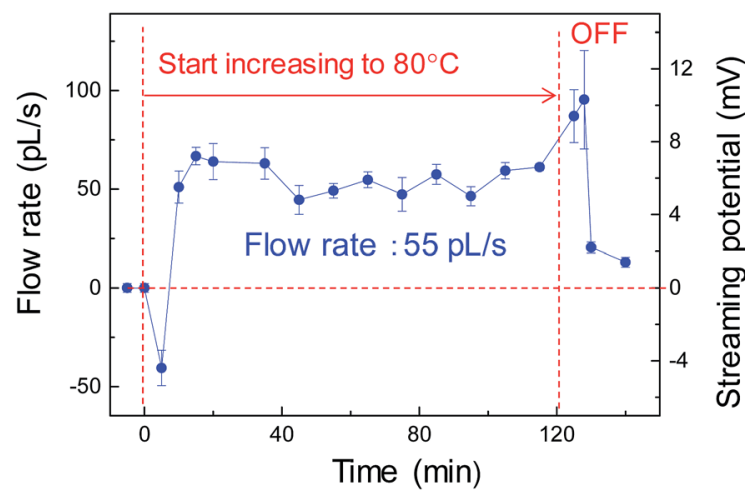

(c)

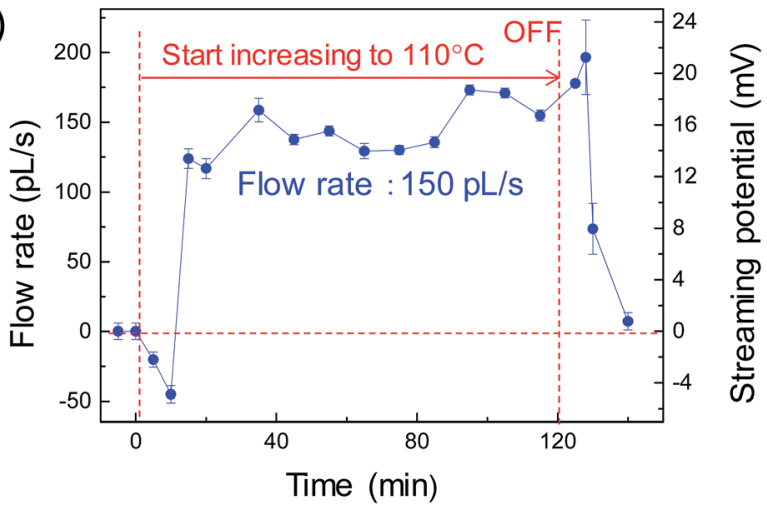

Fig. 6 Verification of the heat-pipe device operation. The water flow rates in the extended nanochannel were monitored by the streaming potential measurements during operation of the heat-pipe device. The evaporator was heated and maintained at (a) $54{ }^{\circ} \mathrm{C}$, (b) $80^{\circ} \mathrm{C}$ and (c) $110^{\circ} \mathrm{C}$ for $120 \mathrm{~min}$ at each temperature.

\subsection{Cooling performance and its improvement}

The cooling performance of the micro heat pipe is roughly estimated by calculating the liquid flow rate $Q \times$ liquid latent heat $L_{\mathrm{m}}$. The maximum flow rate of $150 \mathrm{pL} \mathrm{s}^{-1}$ corresponds to a cooling performance of $3.4 \times 10^{-4} \mathrm{~W} \mathrm{~cm}^{-2}$, which is equivalent to $70 \%$ of the design performance $\left(4.9 \times 10^{-4} \mathrm{~W} \mathrm{~cm}^{-2}\right.$ for the design flow rate of $\left.215 \mathrm{pL} \mathrm{s}^{-1}\right)$. The lower cooling performance than the design value may be due to the high vacuum pressure. Although the vacuum system could evacuate the micro/nanochannel to a gas pressure of $\sim 0.3 \mathrm{kPa}$, the actual gas pressure increased to $\sim 15 \mathrm{kPa}$ before water was introduced. For comparison, the flow rates when the device was operating at

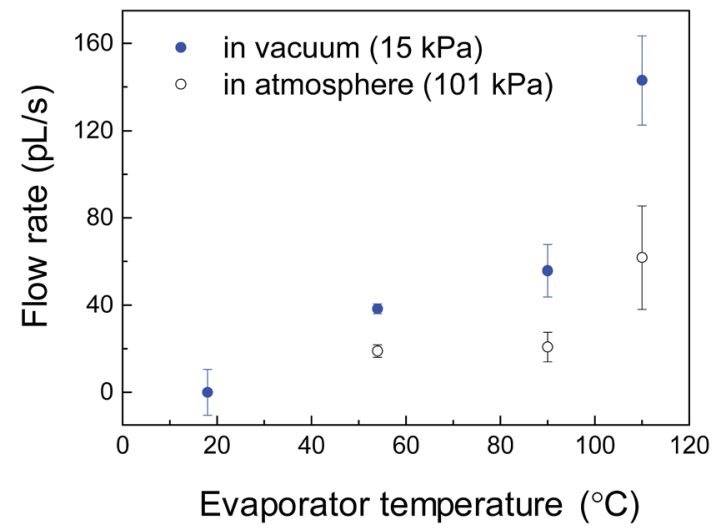

Fig. 7 The water flow rates in the extended nanochannel as a function of evaporator temperature maintained during operation of heat pipe device. Prior to the water charging, the device was evacuated to a vacuum pressure of $\sim 15 \mathrm{kPa}$, or it was maintained at atmospheric pressure $(\sim 101 \mathrm{kPa})$.

atmospheric pressure ( $\sim 101 \mathrm{kPa})$ were measured, as shown in Fig. 6 . The result implies that the cooling performance might be improved using a high-vacuum system.

The low cooling performance is primarily attributed to the very small liquid transport in our current device. Fig. 8 shows a prospective design of a micro heat pipe device for a higher heat transportation. Two sets of side microchannels (with a $7 \mu \mathrm{m}$ depth) are proposed instead of the extended nanochannels (with a $300 \mathrm{~nm}$ depth) for liquid mass transport $\left(\sim 2.5 \mu \mathrm{L} \mathrm{s}^{-1}\right)$. Based on the effect of the surface-to-volume ratio on capillary condensation, high aspect-ratio nanopillars (e.g., nanopillar 1 with a $250 \mathrm{~nm}$ interval and a $7 \mu \mathrm{m}$ depth) are integrated into the device for enhanced capillary condensation. Moreover, an additional set of the extended nanopillar array (e.g., nanopillar 2 with a $250 \mathrm{~nm}$ interval and a $7 \mu \mathrm{m}$ depth) is selectively proposed at the evaporator to mitigate "dry out" for higher operating temperatures and to provide a high Laplace pressure $\left(P_{\mathrm{L}}=1 \mathrm{MPa}\right)$ for driving liquid. The fabrication of these high aspect-ratio nanopillars on a fused silica substrate is challenging, but a similar fabrication process has been

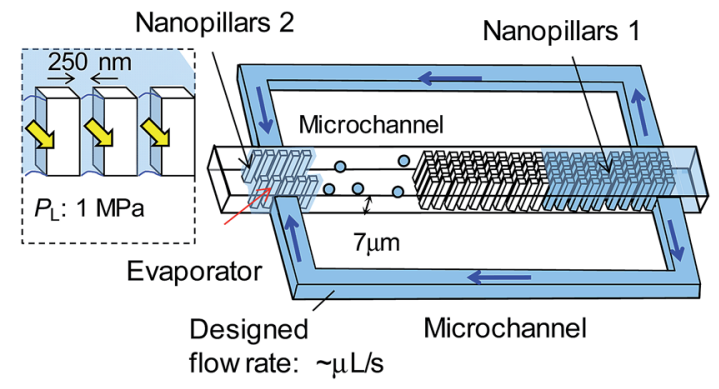

Fig. 8 Prospective design of a micro heat pipe device for a higher heat transportation. High aspect-ratio nanopillars are integrated into the device to drive the mass liquid into the side microchannels $(7 \mu \mathrm{m}$ depth), e.g., nanopillar 1 (a $250 \mathrm{~nm}$ interval and a $7 \mu \mathrm{m}$ depth) for enhanced capillary condensation and nanopillar 2 (a $250 \mathrm{~nm}$ interval and a $7 \mu \mathrm{m}$ depth) for a large Laplace pressure $\left(P_{\mathrm{L}}=1 \mathrm{MPa}\right)$. 
previously described. ${ }^{31}$ The design cooling performance of the prospective device is $2.5 \mathrm{~W} \mathrm{~cm}^{-2}$. Furthermore, tens of these micro heat pipe devices are expected to be integrated on a chip for a highly efficient cooling performance.

\section{Conclusions}

We demonstrated for the first time a two-phase loop micro heat pipe device utilizing extended nanopillars and nanochannels. In contrast to the conventional two-phase loop micro heat pipe device that requires large microchannel and additional wicks, our micro heat pipe device consists of small micro/ nanochannels (containing $\sim 10^{1} \mathrm{~nL}$ liquid) and does not require a wick. The capillary condensation of water on nanopillars was investigated in this study. The experimental results showed a significant enhancement of the condensation rate on nanopillars for a faster vapor-liquid phase transition. In addition, the streaming potential measurement was performed to evaluate liquid transport during operation of the micro heat pipe device, without involving probe molecules. The micro heat pipe device was verified to work properly. Although the cooling performance of the current device was not sufficient, a prospective design of a micro heat pipe device was proposed to realize high-performance cooling. We believe that our micro heat pipe has great potential in thermal control solutions at the chip level for miniaturization of next-generation electronics and optoelectronics.

\section{Conflicts of interest}

There are no conflicts to declare.

\section{Acknowledgements}

This work was financially supported by the Core Research for Evolutional Science and Technology (CREST) of the Science and Technology Corporation (JST) of Japan. The authors acknowledge the financial support from the National Natural Science Foundation of China (Grant No. 51505106), the China Postdoctoral Science Foundation (Grant No. 2017M610207) and the Heilongjiang Postdoctoral Foundation (No. LBH-Z16074).

\section{References}

1 S. M. S. Murshed and C. A. Nieto de Castro, Renewable Sustainable Energy Rev., 2017, 78, 821.

2 J. Qu, H. Wu, P. Cheng, Q. Wang and Q. Sun, Int. J. Heat Mass Transfer, 2017, 110, 294.

3 J. Marschewski, L. Brenner, N. Ebejer, P. Ruch, B. Michel and D. Poulikakos, Energy Environ. Sci., 2017, 10, 780.

4 S. V. Garimella, T. Persoons, J. A. Weibel and V. Gektin, IEEE Trans. Compon., Packag., Manuf. Technol., 2017, 7, 1191.

5 R. Mahajan, C.-P. Chiu and G. Chrysler, Proc. IEEE, 2006, 94, 1476.
6 X. Perpiňà, X. Jordà, M. Vellvehi and J. Altet, Appl. Phys. Lett., 2011, 98, 164104.

7 A. Bar-Cohen and P. Wang, J. Heat Transfer, 2012, 134, 051017.

8 J. B. Marcinichen, J. A. Oliverier, D. De Oliverira and J. R. Thome, Appl. Energy, 2012, 92, 147.

9 K. F. Wiedenheft, H. A. Guo, X. Qu, J. B. Boreyko, F. Liu, K. Zhang, F. Eid, A. Choudhury, Z. Li and C.-H. Chen, Appl. Phys. Lett., 2017, 110, 141601.

10 J. B. Marcinichen, J. A. Oliverier, N. Lamaison and J. R. Thome, Heat Transfer Eng., 2013, 34, 801.

11 N. H. Naqiuddin, L. H. Saw, M. C. Yew, F. Yusof, T. C. Ng and M. K. Yew, Renewable Sustainable Energy Rev., 2018, 82, 901. 12 A. Faghri, J. Heat Transfer, 2012, 134, 123001.

13 H. Behi, M. Ghanbarpour and M. Behi, Appl. Therm. Eng., 2017, 127, 1132.

14 B. Suman, Appl. Mech. Rev., 2007, 60, 107.

15 M. L. Berre, S. Launay, V. Sartre and M. Lallemand, J. Micromech. Microeng., 2003, 13, 436.

16 J. Kirshberg, K. Yerkes, D. Trebotich and D. Liepmann, Am. Soc. Mech. Eng., Micro-Electro-Mech. Syst., 2000, 2, 1-8.

17 C. Wang, T. Leu and T. Lai, Exp. Therm. Fluid Sci., 2008, 32, 1090.

18 K. Mawatari, Y. Kazoe, H. Shimizu, Y. Pihosh and T. Kitamori, Anal. Chem., 2014, 86, 4068.

19 A. Hibara, T. Saito, H. B. Kim, M. Tokeshi, T. Ooi, M. Nakao and T. Kitamori, Anal. Chem., 2002, 74, 6170.

20 T. Tsukahara, A. Hibara, Y. Ikeda and T. Kitamori, Angew. Chem., Int. Ed., 2007, 46, 1180.

21 H. Chinen, K. Mawatari, Y. Pihosh, K. Morikawa, Y. Kazoe, T. Tsukahara and T. Kitamori, Angew. Chem., Int. Ed., 2012, 51, 3573.

22 K. Shirai, K. Mawatari and T. Kitamori, Small, 2014, 10, 1514.

23 R. Ishibashi, K. Mawatari and T. Kitamori, Small, 2012, 8, 1237.

24 Y. Pihosh, J. Uemura, I. Turkevych, K. Mawatari, Y. Kazoe, A. Smirnova and T. Kitamori, Angew. Chem., Int. Ed., 2017, 56, 8130 .

25 A. Hibara, K. Toshin, T. Tsukahara, K. Mawatari and T. Kitamori, Chem. Lett., 2008, 37, 1064.

26 T. Tsukahara, W. Mizutani, K. Mawatari and T. Kitamori, J. Phys. Chem. B, 2009, 113, 10808.

27 Y. Xu, C. Wang, Y. Dong, L. Li, K. Jang, K. Mawatari, T. Suga and T. Kitamrori, Anal. Bioanal. Chem., 2012, 402, 1011.

28 K. Mawatari, T. Tsukahara, Y. Sugii and T. Kitamori, Nanoscale, 2010, 2, 1588.

29 K. Morikawa, K. Mawatari, M. Sato, T. Tsukahara and T. Kitamori, Lab Chip, 2010, 10, 871.

30 K. Morikawa, K. Mawatari, Y. Kazoe, T. Tsukahara and T. Kitamori, Appl. Phys. Lett., 2011, 99, 123115.

31 N. Kaji, Y. Tezuka, Y. Takamura, M. Ueda, T. Nishimoto, H. Nakanishi, Y. Hiriike and Y. Baba, Anal. Chem., 2004, 76, 15. 